\title{
Overview of the Technology of Low Altitude Warning Based on Acoustic Detection
}

\author{
Shaocheng Zhu ${ }^{1, \text { a }}$ and Limin Liu ${ }^{1}$ \\ ${ }^{1}$ Department of Electronic and Optics Engineering, Ordnance Engineering College, Shijiazhuang \\ 050003, China. \\ akolzhu@qq.com
}

Keywords: Low altitude warning, Target Recognition, Acoustic localization

\begin{abstract}
It is very important for the existing warning system to detect low altitude target with acoustic detection due to the blindness of the traditional radar detection. Electronic devices are used in acoustic detection to receive the threat target's sound wave in order to obtain the target's attributes such as coordinates, tracks and categories. Comparing with the traditional warning technology, the warning technology based on acoustic detection has the features of not being to get found easily, to be affected by visibility when being armed. It is especially suitable to defense against the low altitude threat target. Being one of the most popular warning technology, the warning technologies based on acoustic detection has its unique advantages in the field of localization, recognition and other areas, and has attracted more and more attention of the military departments of all countries. In this paper, the warning technology based on acoustic detection is introduced briefly, including basic theory, development history, application in military and shortages. In addition, the localization algorithm with five- element cross array and the theory on acoustic target recognition are discussed in detail, and its utilization prospects are pointed out finally.
\end{abstract}

\section{Introduction}

With the development of radar technology, the detection range and accuracy of radar doubled during the early 21 st century. The active radars are mainly used in the warning system at present. However, the curvature of the earth and the shading of the terrain have great influence on the detection performance of radar. It is difficult to reach the range of elevation angle $0^{\circ} \sim 90^{\circ}$ under the existing conditions. Usually there is a $60^{\circ} \sim 80^{\circ}$ headspace blind and low altitude blind space in traditional [1]. The method of adding high elevation angle wide beam antenna [2] can only increase the complexity and cost, reduce the maneuverability, but cannot solve the problems. Although some radars are developed for low altitude blind spot, such as Pluto radar [3] which used the approach of super-cosecant squared beam [4], it is also difficult to detect close to the ground plane $R\left(0^{\circ}\right)$. In addition, the terrain shielding angle has a greater impact on the detection range, as long as the angle increased slightly, the detection range decline sharply. So it is difficult to detect low altitude target with the traditional radar.

To meet the needs of China's economic and social development, the State Council and the Central Military Commission jointly issued the Opinions on Deepening the Reform of China's Low Altitude Airspace Management [5]. It suggests promoting and deepening the management reform of low altitude airspace and decides to open up airspace below 1000 meters gradually. Helicopters, unmanned aerial vehicles (UAVs), cruise missiles and stealth aircraft fly at low altitude frequently. Their small RCS and short flight time shorten radar's response time and reduce the targets' interception probability. It is difficult for radar to capture air intelligence at low altitude due to the strong electromagnetic interference, complex terrain and meteorological conditions. With these complex conditions, low altitude warning is more difficult.

The technology of low altitude early waring based on acoustic detection detects low altitude air intelligence using the principles of acoustic detection. It compensates the disadvantages of existing 
radar for low altitude warning. It is especially suitable for the detection of acoustic target at low altitude. Thus the capabilities of low altitude reconnaissance and warning will be improved.

\section{Development status}

Acoustic detection technology was born in the First World War, during which the sound detector was used to detect the incoming enemy aircraft and artillery position. It achieved good results in the battlefield. But because of its slow response time and low measurement accuracy, it gradually replaced by radars which are long-term used in the field of air defense warning since then. With the development of IC and IT, acoustic detection technology has re-boarded to the historical stage of air defense warning. The unique advantage of passive detection has won the favor of military departments of all countries and it is successfully applied to underwater and low altitude warning.

\subsection{Foreign development status}

With the increasing complexity of modern electromagnetic environment, there are also some blind areas in radar detection. Acoustic detection technology is used in air defense warning once again due to the unique advantages. With the development of low altitude penetration technology, anti-radiation technology, stealth technology and electronic jamming technology, the United States, Britain, and Israel developed acoustic detection products which can be used to detect and locate the helicopters, UAVs and artilleries.

The United States Dedrone Company has developed a special UAVs detection system [6] to prevent potential terrorist attacks, which uses distributed acoustic and optical sensor to detect UAVs. UAVs can be detected over $80 \mathrm{~m}$. When installed, the system can protect the full airspace with sensors. It warns the illegal invasion of UVAs. What's more, the system updates the feature databank with cloud computing technology timely.

British BAE System Company developed the HALO Mark2 artillery localization system [7]. The system is successful armed in the Balkans, and now available to the British Ministry of Defense Advanced Sound Detection Plan (ASP). The system can detect the coordinate of artillery and mortar using passive acoustic localization technology.

The Search Helicopter acoustic detection system [8] developed by Sweden is equipped with signal processing and display units. It can locate the armed helicopter within $6 \mathrm{~km}$ and determine the flight direction of the helicopter in $4 \mathrm{~s}$. The system can display the coordinate, category and status of the helicopter. It updates every $2 \mathrm{~s}$

Cooperative group TTCP-AG6 [9] which consists of United States, Britain, Canada and Australia proposes to use the 16 elements cross array to detect the low altitude UAVs, the experimental results show that the detection method can undertakes low altitude warning tasks.

\subsection{Domestic development status}

The work of China's low altitude warning based on acoustic detection started later. Human and financial resources are relatively inadequate. We studied the acoustic detection technology from the beginning of 90s. After more than 20 years' development and the support of related research topics, the acoustic detection technology have achieved some corresponding results. There are many institutes achieving good result in acoustic detection. For example, the Institute of Acoustics of the Chinese Academy of Sciences developed acoustic detection and warning network to locate and recognize helicopters, cruise missiles and so on. Nanjing University of Science and Technology studies on Anti-helicopter Mine [10] to defense against low-flying threat helicopters, and Northwestern Polytechnic University studies on the acoustic localization [11] successfully and novel algorithm of passive detection was proposed. 


\subsection{Shortages}

(1) It is difficult to increase the detection range by increasing the size of the sensor network, which directly leads to the limited detection range of the system. Compared with the radar detection range, the acoustic detection system is seriously insufficient.

(2) At present, most of the devices used in sound detection system at home and abroad adopt special electronic devices based on piezoelectric effect, which can be easily suppressed by enemy interference.

(3) It need to lay a special sensor communication network for the acoustic sensor network, which is seriously dependent on energy. It is difficult to achieve wide-area air defense.

\section{Basic theory}

Aircraft flies with noise produced by propulsion system inevitably. Meanwhile, aircraft body noise is produced by the interaction between body surface and air while flying. The modern stealth technology continues to develop, but it is mainly for electromagnetic and infrared stealth. The noise cannot be completely hidden at present. To reduce the noise of fighter aircraft, it is necessary to add additional noise reduction equipment to the propulsion system or change the acoustic characteristics of the existing shape, which will reduce the maneuverability of the fighter and increase the technical complexity. It provides the necessary conditions for the development of low altitude warning technology based on acoustic detection.

Acoustic detection technology is a full passive and omnidirectional detection method. It uses the microphone to receive the sound information from the target, and identifies the target by spectral analysis. What's more, it gets the target position, tracks and attribute information according to the delay and the phase relationship, which produced by the target radiation noise, between the sensors in the sensor array.

\subsection{Acoustic localization}

Accurate positioning of the target focuses on how to determine the direction and distance of target relative to the sensor, which are called direction estimation and distance estimation. Four-element cross array [12], fiver-element cross array [13], planar array and three-dimensional array [14] are mainly used to detect. These arrays are mainly utilized to estimate the azimuth and distance of the target using the delay time of the target sound source to among acoustic sensors in the array. So if we can accurately estimate the time delay, and then according to the geometric relationship between the microphone arrays, we can calculate the parameters of the target position estimation. In this paper, a five-element cross array [15] is introduced to illustrate the localization algorithm.

The five-element cross-array consists of five identical acoustic sensors $S_{0}, S_{1}, S_{2}, S_{3}, S_{4}$ spaced on the $X$-Y plane, with four sensors $S_{1}, S_{2}, S_{3}, S_{4}$ evenly on a ring of radius $D$ and $S_{0}$ at the origin of the Cartesian coordinate system, as shown in the Fig.1. Let the target $T(x, y, z)$ be a point source and propagate in the form of a spherical wave.

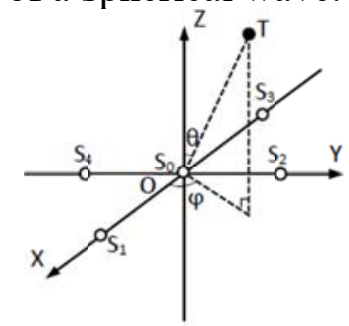

Fig.1 The five-element cross acoustic array

The range $r$ is the distance between the target and the origin. The range $r_{i}$ is the distance between the target and the $i$ th $s_{i}$ sensor, the target azimuth angle is $\varphi$, defined counterclockwise relative to the $X$-axis, and the target elevation angle is $\theta$, defined clockwise relative to the $Z$-axis. 
$\tau_{i}(i=1,2,3,4)$ is the signal delay between the $i$ th $s_{i}$ sensor and the origin sensor $s_{0}$, c denotes the velocity of sound in the air, $c=340 \mathrm{~m} / \mathrm{s}$.

$$
\tau_{i}=\frac{r_{i}-r}{c}
$$

Considering the geometrical of the target and the acoustic array, we have the following relations respectively.

$$
\left\{\begin{array}{l}
D^{2}+2 r c \tau_{1}-\left(c \tau_{1}\right)^{2}-2 r D \sin \theta \cos \varphi=0 \\
D^{2}+2 r c \tau_{2}-\left(c \tau_{2}\right)^{2}-2 r D \sin \theta \sin \varphi=0 \\
D^{2}+2 r c \tau_{3}-\left(c \tau_{3}\right)^{2}+2 r D \sin \theta \cos \varphi=0 \\
D^{2}+2 r c \tau_{4}-\left(c \tau_{4}\right)^{2}+2 r D \sin \theta \sin \varphi=0
\end{array}\right.
$$

Summing the Eqs. (2a)-(2d),we have the following target location equations.

and

$$
\begin{gathered}
r=\frac{\left(c^{2} \sum_{i=1}^{4} \tau_{i}^{2}-4 D^{2}\right)}{\left(2 c \sum_{i=1}^{4} \tau_{i}\right)} \\
\varphi=\arctan \left(\frac{\tau_{2}-\tau_{4}}{\tau_{1}-\tau_{3}}\right)
\end{gathered}
$$

$$
\theta=\arcsin \left(\frac{c \times \sqrt{\left(\tau_{1}-\tau_{3}\right)^{2}-\left(\tau_{2}-\tau_{4}\right)^{2}}}{D}\right)
$$

Eqs. (3)-(5) show that we can locate the low altitude acoustic target of interest accurately by the Time Delay of Arrival (TDOA) [14]. Nowadays, time delay estimation algorithms mainly include generalized cross correlation algorithm (GCC) [16], time-delay estimation based on spectrum [17], and delay estimation algorithm based on higher order cumulant [18] and so on.

\subsection{Acoustic target recognition}

Target recognition is one of the most important tasks of the warning. Target recognition belongs to the category of pattern recognition. It takes the d-dimensional feature column vector $\boldsymbol{x}$ as input which is the characteristic of the observed acoustic signal. The real number $y$ is the output of the function $y=f(\boldsymbol{x})$, and $\boldsymbol{x}$ is determined by $y$ to be the class of $\omega_{1}, \omega_{2}, \cdots, \omega_{m}$. Normally the sample $\boldsymbol{x}_{i}(i=1,2, \cdots, n)$ consists of $n$ input samples. Typical target recognition system usually consists of the following components: signal acquisition, signal pre-processing, feature extraction and classifier design, as shown in Fig.2.

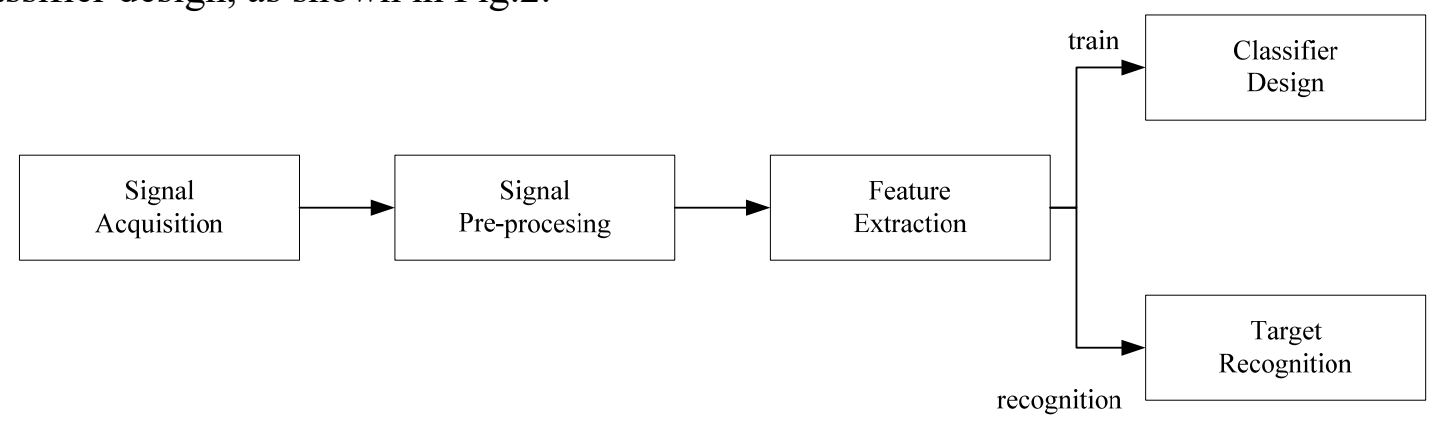

Fig.2 Schematic diagram of acoustic target recognition

Signal acquisition is the process which use acoustic sensors to sample the acoustic signal from the target of interest and then quantify and code the signal. Pre-processing is to detect abnormal data [19] and de-noise for the input sample data before feature extraction to improve the SNR. The suitable de-noising algorithm can effectively remove the influence of natural background colored noise on the target acoustic signal and improve the robustness and accuracy of the target recognition system. There are many typical de-noising algorithm, such as wavelet de-noising [20], spectral subtraction de-noising [21], de-noising based on the independent analysis [22], de-noising based on empirical mode decomposition [23], and de-noising based on the blind source separation [24], etc. 
Feature extraction is a key step in the recognition system, which is to extract the feature vector from the pre-processed signal that contains the acoustic target characteristic. It is also regarded as a projection of the original data space to the feature space, which requires large dispersion between classes, small dispersion within the class. There are many excellent algorithm, such as frequency domain features [25], parametric model features [26], time-frequency features [27], auditory perceptual features [28] and visual features [29], etc. The classifier is designed to classify rules so that the recognition rate of the target is minimized by this rule. Neighborhood [30], neural network [31], support vector machine [32] and hidden Markov model [33] are commonly used.

\section{Development tendency}

Modern war is information-led war, timely and accurate warning directly affects the system of information rights. The low altitude warning technology based on acoustic detection has the unique advantage in combat because of its nature. Some weapons fail directly due to the strong electromagnetic interference in the battlefield. However, acoustic sensors are not affected by it. It provide a new way to detect and warn for low altitude target which makes up for the blind space of the existing air defense warning system. Therefore acoustic detection equipment is essential to future battlefield, it should improve detection equipment performance and enrich their species from the practical point.

(1) The core of the warning is the discovering the target timely and accurately, which requires the acoustic sensor should have a wide frequency response range, small distortion, dynamic range, high sensitivity, and anti-noise ability. But the existing acoustic sensors are not sufficient to meet the above requirements. A new type of acoustic sensors should be vigorously developed with high performance.

(2) The number of acoustic sensors and array layout directly affect the positioning accuracy of the target. In order to maximize the positioning accuracy and the range, it should be developed from the existing layout mode to the stereo cross-over random array. The coordination and performance complementation between sensors in the array can overcome the disadvantages of the simple one sensor through the integration of multiple acoustic sensors.

(3) The acoustic detection sensor is easily affected by the natural background noise, which greatly reduces the detection range, detection accuracy and target recognition performance. In order to guarantee the system's robustness, we can reduce the influence of background noise from hardware and algorithm.

(4) To achieve full airspace, full frequency, and multi-target low altitude warning, it can integrate a variety of detection methods, which complement each other to make up for the detection of vulnerabilities.

\section{Conclusion}

In this paper, the low altitude warning based on acoustic detection is introduced in detail, the basic concepts and the problems are discussed. At the same time, the present and future potential values are introduced. With its development, the solution to the problem is not fully worked out, and there is still a gap between the actual utilization and the theory. But its potential application in the field of air defense warning value for all to see.

\section{References}

[1] Li N. Design Consideration of Air Surveillance Radar's Coverage [J]. Radar Science and Technology, 2005, 3(3): 129-135.

[2] Shen J, Hu B. Application of the Acoustic Sensor Wireless Network in the Blind Compensation of Radar for Tactical Air Defense [J]. Sensor World, 2009, 15(8): 39-42.

[3] Chai Z. View on Foreign Radar Technology Development [J]. Modern Defense Technology, 2004, 32(5): 62-65+68. 
[4] Huang C, Li Z. Development of Dourly Reflector with Extra Cosecant Square Shaped Beam and Low Side-lobe for S-band Target Searching Radar [J]. Modern Radar, 1997, 19(6): 35-50.

[5] Xia Q. Deepening the Reform of China's Low Altitude Airspace Management [N]. 2010.11.15.

[6] Jin C, Pang Z, Hou R. Application of Acoustic Detection Technology in Reconnaissance Warning Equipments [M]. Springer Singapore, 2016.

[7] Feng J, Yang B, Li Z. Overview of Acoustic Detection and Its Application in Military [J]. Audio Engineering, 2012, 36(6): 32-36.

[8] Tang H, Zhao D, Zhang H. The Construction of Anti-air Network System with All-Fiber Passive Acoustic Defense Technology [J]. Chinese Journal of Nature, 2010, 32(1): 40-45.

[9] Pham T, Sour N. TTCP AG-6: Acoustic Detection and Tracking of UAVs; proceedings of the Defense and Security, F, 2004 [C].

[10] Xu K. Research on Several Key Technologies of Passive Acoustic Direction [D]; Nanjing University of Science and Technology, 2005.

[11] Chen H. On Passive Acoustic Direction Finding for Low Altitude Targets [D]; Northwestern Polytechnic University, 2004.

[12] Xiang J. Research on Passive Acoustic Localization Based on Four Cross Array [D]; North Central University, 2008.

[13] Chen H, Zhao J. On Locating Low Altitude Moving Targets Using a Planar Acoustic Sensor Array [J]. Applied Acoustics, 2003, 64(11): 1087-1101.

[14] Gao G. Study on Coordinate Measurement Technology of Impact Points Based on Acoustic Detection [J]. Science Technology and Engineering, 2016, 16(13): 230-234.

[15] Zhang W, Gong C, Sun H. Beamforming Analysis of Five-element Plane Cross Array of Passive Acoustic Detection Technology; proceedings of the National Conference on Acoustics, Chinese Acoustics Society, 2014, Nanjing, Jiangsu Province China, F, 2014 [C].

[16] Li J, Zhao Y, Li D. Accurate Single-observer Passive Coherent Location Estimation Based on TDOA and DOA [J]. Chinese Journal of Aeronautics, 2014, 27(4): 913-923.

[17] Wu F. Research on TOA/TDOA Parameters Estimation Methods for Wireless Location [D]; Jilin University 2006.

[18] Li Y. Research on the TDOA Estimation of OFDM System [D]; University of Electronic Science and Technology, 2014.

[19] Peter H. Machine Learning in Action [M]. Manning Publications Co., 2012.

[20] Quan F, Gu X, Lv Y. Research on De-noising Method of Battlefield Acoustic Signal Based on Wavelet Transform [J]. Computer Knowledge and Technology, 2010, 6(4): 939-941.

[21] Zhang Y, Lin J, Guo L. Study of Birds Sound Classification Based on ABAP Multi band Spectral Subtraction [J]. Journal of Xinyang Normal University (Natural Science Edition), 2015, 28(1): 127-132.

[22] Yang X, Zhang X, Li B. A De-noising Method of Acoustic Signals Based on Kernel Independent Component Analysis [J]. Transducer and Microsystem Technologies, 2011, 30(11): 43-45+48.

[23] Gao Y, Sang E, Liu B. An Adaptive De-noising Algorithm Based on Empirical Mode Decomposition [J]. Computer Engineering and Application, 2007, 43(26): 59-61.

[24] Huang C, Ye J, Pan Z. Application Blind Source Separation Approach for Interference and De-noise within Vibration Signal of Radar [J]. Journal of Anhui University of Technology (Natural Science), 2007, 24(2): 191-194.

[25] Chen R. The Research on Feature Extraction of Target Identification Underwater [D]; South China University of Technology, 2011.

[26] Shen Z. Research on Feature Extraction of Target Acoustic Signals in Complex Background and Its Key Techniques [D]; North Central University, 2010.

[27] Li H, Cheng Y, Dai W. A Method Based on Wavelet Fractal and Support Vector Machine for Underwater Target Recognition [J]. Technical Acoustics 2015, 34(3): 219-222.

[28] Tao B, Guo Q, Miao F. SoC Design of Voiceprint Feature Extraction Based on Improved Mel Filter Banks [J]. Microelectronics, 2015, 40(6): 785-788. 
[29] Zeng X. Intelligent Water Target Recognition [M]. Beijing: National Defense Industry Press, 2016.3 .

[30] Song L, Luo J. Pattern Recognition [M]. Beijing: Machinery Industry Press, 2015.8.

[31] Liu Y, Yu Y, Meng C. Design Research of an Acoustic Target Recognition System Based on Particle Swarm Neural Network [J]. Electric Automation, 2016, 38(2): 115-118.

[32] Li J, Xu J, Li H. Research on Helicopter Target Identification based on Support Vector Machine [J]. Fire Control and Command Control, 2008, 33(1): 31-34.

[33] Zhang Y, Yang J, Sun D. Application of Wavelet MFCC and HMM in Low Altitude Acoustic Target Recognition [J]. Information and Electronic Engineering, 2011, 9(06): 744-748. 